\title{
Fachliche Qualifikation medizinischer Sachverständiger
}

Fritz Dolder

* Teil 1 ist in SÄZ 28/2001 erschienen: Dolder F. Medizinische Gutachten im Kunstfehlerprozess. Ablehnung medizinischer Sachverständiger wegen Befangenheit. Schweiz Arztezeitung 2001;82(28):1525-29.

Korrespondenz:

Prof. Dr. Dr. Fritz Dolder

Universität Basel

Juristische Fakultät

Maiengasse 51

$\mathrm{CH}-4056$ Basel

\section{Gesetzliche Rahmenbedingungen der Gutachtertätigkeit im Kunstfehlerprozess} Bekanntlich werden Kunstfehlerprozesse von medizinischen Sachverständigen entschieden. Deren Beitrag an der Entscheidungsfindung überwiegt regelmässig die Beiträge der Richter und der Prozessparteien um ein Vielfaches. Deshalb erhalten gemäss Bundeszivilprozessgesetz (und einigen kantonalen Zivilprozessgesetzen) die am Verfahren beteiligten Parteien Gelegenheit, vor der Ernennung von Sachverständigen Einwendungen gegen die in Aussicht genommenen Personen vorzubringen. Im Bundesstrafprozess ist vorgesehen, dass der Richter einen oder mehrere Sachverständige ernennt und ihre Namen den Parteien mitteilt. Dies kann nur bedeuten, dass die Parteien nach der Bekanntgabe der Namen ebenfalls Einwendungen gegen die in Aussicht genommenen bzw. bereits ernannten Sachverständigen vorbringen können. Es wird deshalb auch im Bereich des Strafprozesses generell empfohlen, vor der Bestellung von Sachverständigen ein Einvernehmen mit den Prozessparteien zur Person des Gutachters zu erzielen und nur Sachverständige zu ernennen, die das Vertrauen beider Parteien geniessen, um nicht nachträgliche Einwendungen zu riskieren (so z. B. Schmid [1], Hauser und Schweri [2] und Staub [3]).

In Verfahren zur Beurteilung ärztlicher Kunstfehler fragt es sich, ob derartige Einwendungen der Prozessparteien gegen einen in Aussicht genommenen Gutachter sich auch auf dessen fachliche Qualifikation beziehen dürfen - neben den klassischen Ablehnungsgründen wie z.B. Befangenheit aufgrund von persönlichen Beziehungen.

\section{Der Sachverhalt}

Im Rahmen eines Kunstfehlerprozesses (Strafverfahren) waren die therapeutische Wirksamkeit und unerwünschten Nebenwirkungen eines Medikamentes zur Therapie der koronaren Herzkrankheit, insbesondere zur Reinfarzierungsprophylaxe, zu beurteilen. Die Untersuchungsbehörde versuchte zunächst, den Direktor des Instituts für Rechtsmedizin der grössten medizinischen Fakultät der Schweiz für die Ausarbeitung eines Gutachtens zu gewinnen. Der ange- fragte Professor lehnte indessen schon nach wenigen Tagen ab; das Aktenstudium und die aufgeworfenen Fragen «hätten ihn eindeutig zum Schluss geführt, dass ein klinisch tätiger Fachgutachter, am besten ein versierter klinisch-pharmakotherapeutisch tätiger Internist, den Gutachterauftrag übernehmen solle».

Trotz dieser deutlichen Empfehlung beauftragte die Untersuchungsbehörde daraufhin einen Rechtsmediziner einer anderen Universität mit der Ausarbeitung eines medizinischen Gutachtens. Dessen medizinische Biographie wies nach eigenen Angaben folgende Stationen auf: Nach Abschluss des Medizinstudiums und Militärdienstes wissenschaftlicher Angestellter am Institut für Rechtsmedizin einer Universität, Qualifizierung und praktische Tätigkeit als Facharzt für Rechtsmedizin; Oberarzt an einer Psychiatrischen Universitätsklinik, Qualifizierung als Facharzt für Psychiatrie; leitender Arzt für forensische Psychiatrie an einer Psychiatrischen Uniklinik und Habilitation für forensische Psychiatrie.

Vor Bundesgericht war zu beurteilen, ob der Anspruch des Geschädigten auf den Ausstand Befangener und auf rechtmässige Zusammensetzung der entscheidenden Behörde dadurch verletzt worden sei, dass diese Behörde ausschliesslich auf das Gutachten des betreffenden Gutachters abgestellt hatte.

\section{Aus der Urteilsbegründung}

2.b) [...] Der Beschwerdeführer rügt aber, der von der Staatsanwaltschaft beauftragte unabhängige Gutachter, Y., sei nicht sachkundig. Y. sei Psychiater und Gerichtsmediziner und daher nicht der richtige Fachmann zur Beurteilung der Wirksamkeit eines Kardiologiemedikamentes. Genügend sachkundig wäre nach Auffassung des Beschwerdeführers allein ein versierter klinisch-pharmakotherapeutisch tätiger Internist gewesen. Der Anspruch auf rechtmässige Zusammensetzung der entscheidenden Behörde und den Ausstand Befangener umfasse auch einen Anspruch darauf, dass der eingesetzte Sachverständige über die im Einzelfall erforderliche Sachkunde verfüge. Dieser Anspruch sei von den kantonalen Behörden verletzt worden. 
Für Sachverständige gelten [...] grundsätzlich die gleichen Ausstands- und Ablehnungsgründe, wie sie für den Richter vorgesehen sind. Es rechtfertigt sich daher, die Rechtsprechung zur Verfahrensgarantie [...], soweit es um die richterliche Unabhängigkeit und Unparteilichkeit geht, sinngemäss auf das Erfordernis der Unabhängigkeit und Unparteilichkeit des Sachverständigen anzuwenden. Danach ist Befangenheit anzunehmen, wenn Umstände vorliegen, die geeignet sind, Misstrauen in die Unparteilichkeit eines Sachverständigen zu erwecken [...]. Als besondere Umstände, die Misstrauen in die Unparteilichkeit eines Sachverständigen erwecken, sind grundsätzlich dieselben Umstände zu verstehen, die auch Misstrauen in die Unparteilichkeit eines Richters wecken würden. Die fehlende Sachkunde eines Gutachters bildet keinen solchen Umstand, sondern ist bei der Würdigung des Gutachtens zu berücksichtigen (vgl. Donatsch [4]). Ergibt sich nach Erstattung des Gutachtens, dass dem Gutachter die erforderliche Sachkunde fehlte, darf das Gericht auf sein Gutachten nicht abstellen. Die Frage, ob der beigezogene Gutachter sachkundig ist oder nicht, betrifft daher die Beweiswürdigung und ist materieller, nicht formeller Natur. [...].

\section{Zum Kontrast}

Aus einem Beschluss des Bezirksgerichts Meilen in einem anderen Kunstfehlerprozess (Zivilrecht); Z08/CG970028 vom 30. Juni 1999 (rechtskräftig):

1.a) Gemäss $\$ 172$ Abs. 2 ZPO erhalten die Parteien Gelegenheit, gegen die Ernennung der Sachverständigen Einwendungen zu erheben. Die Einwendungen können die fachliche Eignung oder Ausstandsgründe betreffen und müssen begründet sein (Frank/Sträuli/Messmer, Kommentar zur zürcherischen Zivilprozessordnung, 3. Aufl., Zürich 1997, $\S 172$ N 3). [...].

\section{Kommentar}

Nach einhelliger Bekundung aus medizinischen Kreisen gibt es in der Schweiz Qualitätsprobleme im Bereich der medizinischen Begutachtung (z.B. Bär und Meine [5] und Meine [6]). Die Rechtsprechung sollte dieser Problematik dadurch Rechnung tragen, dass sie an die Qualität der medizinischen Sachverständigen und ihrer Gutachten tendenziell hohe Anforderungen stellt.

Das referierte Urteil des Bundesgerichts anerkennt zwar, dass die Erstattung eines Gutachtens durch einen nicht ausreichend qualifizierten Sachverständigen einen Fehler darstellt, der nachträglich bei der Würdigung des Gutachtens berücksichtigt werden kann. Leider geht das Urteil des Bundesgerichts aber nicht weiter: Es stuft die fehlende Qualifikation nicht als einen Ablehnungsgrund ein, der es den Prozessparteien (Arzt, Geschädigter) erlauben würde, die fehlende Qualifikation des Sachverständigen bereits zu einem Zeitpunkt zu rügen, bevor dieser seine Arbeit an dem Gutachten aufnimmt. Diese Unterscheidung hat im referierten Fall dazu geführt, dass das Bundesgericht die Rüge der fehlenden Qualifikation des Sachverständigen nicht als Frage der verfassungsmässigen Zusammensetzung der entscheidenden Behörde eingestuft hat und deshalb auf die Beschwerde insoweit gar nicht eingetreten ist.

Diese Haltung erscheint zumindest als realitätsfern: Wie bereits früher in der SÄZ mit Bezug auf Gutachten hervorgehoben worden ist, rechnet sie «nicht genügend mit der menschlichen Natur: [...] Befunde und Beurteilungen, die einmal in den Akten stehen, drohen doch nach aller Erfahrung ihre Wirkung auch dann zu entfalten, wenn sie einer wirklich neutralen Beurteilung nicht standhalten würden.» [7]. In Umkehrung eines bekannten Satzes sollte deshalb davon ausgegangen werden, dass ein anderer Zusammenhang gilt: Einmal in den Akten, immer im Urteil: Was einmal in den Prozessakten steht, bleibt in den Köpfen der Richter und wirkt sich immer auch auf das Urteil aus, selbst wenn ein Gutachten später aus irgendwelchen Gründen aus den Akten entfernt worden ist. Aus diesem Grund sollte den Prozessparteien ein Instrument zur Verfügung stehen, um einen Sachverständigen ohne ausreichende Qualifikation bereits vor der Aufnahme seiner für das Prozessergebnis regelmässig entscheidenden Arbeit zu eliminieren.

Nachträgliche Kontrolle des Gutachtens? Das Urteil verweist auf eine nachträgliche Kontrolle des von einem nichtqualifizierten Sachverständigen erstatteten Gutachtens: Ergebe sich nach Erstattung des Gutachtens, dass dem Gutachter die erforderliche Qualifikation fehlte, dürfe das Gericht auf sein Gutachten nicht abstellen. Das Urteil (und die zitierte Kommentarstelle) überschätzt dabei leider die praktischen Möglichkeiten von Richtern und Gerichten.

Ausgangspunkt für den Einsatz eines medizinischen Sachverständigen ist eine Entscheidungssituation, in der der Richter nicht sachkundig ist: deshalb - und nur deshalb - braucht er einen Sachverständigen. Wenn dem aber so ist, wie soll der arme, fachlich überforderte Richter jetzt plötzlich genügend sachkundig sein, um ein - regelmässig kompliziertes und häufig sogar für Fachleute schwer verständliches - «schlechtes» Gutachten eines nichtqualifizierten Sachverständigen zu erkennen und von einem «guten» 
Gutachten eines qualifizierten Sachverständigen zu unterscheiden? Dazu bedarf es doch erst recht der wissenschaftlichen Sachkunde, die dem Richter fehlt! In der Praxis ist der Richter erfahrungsgemäss einem einmal ernannten Sachverständigen auf Gedeih und Verderb ausgeliefert und ist jedenfalls nicht ohne fremde Hilfe in der Lage, die wissenschaftliche Qualität eines Gutachtens zu beurteilen.

Auch aus diesem Grund sollte den Prozessparteien ein Instrument zur Verfügung stehen, welches erlaubt, einen nicht ausreichend qualifizierten Sachverständigen schon vor Aufnahme seiner Arbeit zu eliminieren.

Bereits der Hinweis im zivilrechtlichen Kontrasturteil eines zürcherischen Bezirksgerichts zeigt dafür einen möglichen Weg auf: Da sich die Prozessgesetze durchwegs des Begriffs des Sachverständigen bedienen, werden sie verletzt, wenn ein Nichtsachverständiger ernannt wird, und zwar auch dann, wenn das fragliche Gesetz die fehlende fachliche Qualifikation nicht ausdrücklich als Ablehnungsgrund nennt. Die Beauftragung eines Nichtsachverständigen könnte daher von den Prozessparteien möglicherweise mit einem prozessualen Rechtsmittel (Rekurs, Beschwerde o.ä.) gerügt werden, bevor der Ernannte seine Arbeit als Gutachter aufnimmt. Allerdings setzt dies voraus, dass den Prozessparteien die Ernennung des Sachverständigen wenigstens rechtzeitig, d. h. vor Beginn der Arbeiten an dem Gutachten, mitgeteilt wird.

Im Gegensatz zu der vom Bundesgericht zitierten Kommentarstelle scheint sich im Zivilprozess (und auch im Sozialversicherungsrecht) allmählich die Ansicht durchzusetzen, dass die fehlende Qualifikation eines medizinischen Gutachters einen Umstand darstellt, der bereits vor der Beauftragung gerügt werden kann und bereits zu diesem Zeitpunkt zur Eliminierung der betreffenden Person vor Erstattung des Gutachtens führen kann (so nicht nur das zitierte Kontrasturteil des Bezirksgerichts Meilen und der bereits zitierte Aufsatz von Kuhn [7], sondern auch Leuch, Marbach, Kellerhals und Sterchi [8], und Bühler, Edelmann und Killer [9]). Und es ist überhaupt nicht einzusehen, warum für die Begutachtung in einem Strafverfahren eine geringere Qualifikation eines Sachverständigen genügen sollte als in einem Zivilprozess.

\section{Qualifikation von medizinischen Sachverständigen}

Was gehört nun zu einer ausreichenden Qualifikation eines medizinischen Sachverständigen in einem Kunstfehlerprozess, also in Verfahren, in denen die Qualität der Berufsarbeit von Ärzten beurteilt wird? Zur Beantwortung dieser Frage sollte gerade aufgrund der Erfahrungen des referierten Bundesgerichtsurteils in erster Linie auf praktische und weniger auf formelle Kriterien abgestellt werden:

Sachverständig für die Beurteilung von möglichen ärztlichen Kunstfehlern ist, wer in seiner täglichen und aktuellen Berufstätigkeit die gleichen (diagnostischen, therapeutischen) Aktivitäten, welche er in seinem Gutachten zu beurteilen hat, regelmässig (täglich, mindestens aber: wöchentlich) und erfolgreich ausführt.

Diese Forderung wird nicht immer leicht zu erfüllen sein; insbesondere wird es in vielen Fällen noch nicht generell genügen, dass Gutachter und beurteilter Arzt die Ausbildung zum gleichen Spezialarzt FMH aufweisen. Vielmehr sollte es darüber hinaus entscheidend auf die praktische klinische Tätigkeit ankommen, weil nur diese das Augenmass und die handwerkliche Erfahrung für die kritische Beurteilung klinischer Notwendigkeiten in ihrem ständigen Spannungsfeld zu den klinischen Möglichkeiten vermittelt. Allerdings wächst gerade bei zunehmender Nähe der klinischen Tätigkeit von Gutachter und beurteiltem Arzt auch das Risiko, dass zwischen ihnen bereits persönliche Beziehungen bestehen, welche zur Ablehnung des Gutachters aus den klassischen Gründen der Befangenheit führen können. Selbstverständlich werden auch nicht alle interessierten Kreise an diesem Vorschlag Freude haben, namentlich nicht Ärzte im Ruhestand, welche nicht mehr klinisch, aber als Gutachter tätig sind.

Leider ist auch zu befürchten, dass sich formale Gesichtspunkte bei der Beurteilung der Gutachterqualifikation nur schwerlich völlig eliminieren lassen; sie dürften dann wohl zwangsläufig in die bekannten Elemente eines peer review (mit den bekannten Besonderheiten der sogenannten Krähenoptik) ausmünden: Ein Spezialarzt FMH wird sich vermutlich nur ungern von einem Nicht-Spezialarzt FMH beurteilen lassen, ein Professor ungern von einem Nicht-Professor usw. Dies führt weiter zu einer zweiten, generellen ImZweifel-Regel (in diesem Sinne schon Staub [3]):

Bestehen Zweifel hinsichtlich der Qualifikation eines Gutachters, so sollte dieser nicht ernannt werden; Gutachten sollten nur an Personen vergeben werden, deren Qualifikation zur Beurteilung des betreffenden Einzelfalles ausser jedem Zweifel steht.

Vor dem Hintergrund dieser beiden Vorschläge sollte auch die Qualifikation von Rechtsmedizinern zur Beurteilung von ärztlichen Kunstfehlern mit kritischer Zurückhaltung beurteilt werden. So wurde in der deutschen Praxis die generelle Emp- 
fehlung ausgesprochen, dass für die Prüfung, ob ein Schaden auf einen medizinischen Berufsfehler zurückzuführen sei, vom Rechtsmediziner prinzipiell die Hinzuziehung eines weiteren Sachverständigen aus dem betreffenden klinischen Fachgebiet empfohlen werden solle. Grundsätzlich solle der gerichtsmedizinische Gutachter seine Kompetenz kritisch prüfen und, wenn diese nicht sicher ausreiche und er nur noch «Bücherwissen» anbieten könne, die Hinzuziehung eines weiteren Sachverständigen aus dem betreffenden Fachgebiet empfehlen [10].

Mit diesen Hinweisen schliesst sich der Kreis zu dem hier referierten Bundesgerichtsurteil: Dass der betreffende Rechtsmediziner ausschliesslich «Bücherwissen» für die Beurteilung der gestellten klinischen Fragen anbieten konnte, war unbestritten. Die wissenschaftliche Qualität des Gutachtens ergab sich glücklicherweise schon bald nach dem Urteil des Bundesgerichts: Das fragliche Medikament - dessen therapeutische Wirksamkeit und Unbedenklichkeit das Gutachten vehement verteidigt hatte - wurde ein knappes halbes Jahr nach dem Urteil aufgrund erwiesener Unwirksamkeit und gefährlicher Nebenwirkungen (coronary steal effect) vom deutschen Markt und wenig später auch vom schweizerischen Markt zurückgenommen. Dass dieser Rückzug erst rund dreissig (!) Jahre nach den ersten kritischen Publikationen über das fragliche Medikament in der wissenschaftlichen Literatur erfolgte, weckt allerdings Zweifel an den Möglichkeiten der staatlichen (nicht nur schweizerischen) Medikamentenkontrolle.

(Urteil des Bundesgerichts 1P.553/1999)

\section{Literatur}

1 Schmid N. Strafprozeßrecht. 2. Auflage. Zürich: Schulthess; 1993. Nr. 666.

2 Hauser R., Schweri E. Schweizerisches Strafprozessrecht. 5. Auflage. Basel: Helbing \& Lichtenhahn; 2002. § 64 / Nr. 6.

3 Staub P. Kommentar zum Strafverfahren des Kantons Bern. Bern: Paul Haupt; 1993. N. 1 zu Art. 151.

4 Donatsch A. N. 28 zu § 111. In: Donatsch A, Schmid N. Kommentar zur Strafprozessordnung des Kantons Zürich vom 4. Mai 1919. Zürich: Schulthess; 2000.

5 Bär E, Meine J. Gutachternotstand in der Schweiz. Schweiz Ärztezeitung 1998;79(7):240-1.

6 Meine J. L'expertise médicale en Suisse: satisfait-elle aux exigences de qualité actuelles? SVZ 1999;67:37; deutscher Text: Meine J. Die ärztliche Unfallbegutachtung in der Schweiz - Erfüllt sie die heutigen Qualitätsanforderungen? Swiss Surg 1998;4(2):53-7.

7 Kuhn HP. SUVA darf «fremde» Gutachten verwenden. Schweiz Ärztezeitung 1999;80(44):2581-2.

8 Leuch G, Marbach O, Kellerhals F, Sterchi M. Die Zivilprozessordnung für den Kanton Bern. 5. Auflage. Bern : Stämpfli; 2000. N. 1a zu Art. 265, N. 1 b zu Art. 267.

9 Bühler A, Edelmann A, Killer A. Kommentar zur aargauischen Zivilprozessordnung. Zürich: Schulthess; 1998. N. 7 zu § 254.

10 Janssen W, Püschel K. Zur Frage der GutachterKompetenz in der Beurteilung ärztlicher Behandlungsfehler (sog. Kunstfehler). Med Recht 1998; 16(3):119-21. 